\title{
Photosensitization in Cattle Caused by Spontaneous and Experimentally Ingestion of Stryphnodendron fissuratum
}

\author{
Neylisa Dario Lazaro' ${ }^{1}$, Flavia Barbieri Bacha ${ }^{2}$, Rayane Chitolina Pupin ${ }^{3}$, Juliana Paniago Lordello de Paula ${ }^{3}$, \\ Paula Velozo Leal ${ }^{3}$, Arnildo Pott ${ }^{4}$, Danilo Carloto Gomes ${ }^{5}$ \& Ricardo Antônio Amaral de Lemos ${ }^{5}$
}

Background: Stryphnodendron fissuratum is a tree from the Brazilian Cerrado. Its fruit is toxic to cattle and can cause clinical digestive signs, hepatogenous photosensitization, and abortion. Cases of poisoning in cattle, goats and guinea pigs have been experimentally reproduced; however, photosensitization could not be reproduced. The aim of this work was to describe an outbreak of natural poisoning and experimental reproduction in cattle, both with hepatogenous photosensitization. Materials, Methods \& Results: Its described and natural outbreak and an experimental poisoning. In the outbreak, three bovines in the acute phase and three in the chronic phase were examined. Blood samples were collected from all of these animals in order to measure serum levels of aspartate aminotransferase (AST), gamma-glutamyl transferase (GGT), urea, and creatinine. The first three animals underwent necropsy and histopathological evaluation. The experiment was conducted with two nine-month-old calves that received an oral paste made with crushed $S$. fissuratum fruits mixed with water. These fruits were collected at a farm at which cattle poisoning cases had occurred. Blood samples were collected in order to measure serum levels of AST, GGT, urea, and creatinine, before plant administration and then daily during the experimental period. Skin biopsies were taken before plant administration and new one after the first signs of skin lesions. The natural outbreak affected 52 of 160 bovine ( 31 calves and 21 cows) in the lot. Two calves and 14 cows died. Clinical signs consisted of depression, ataxia, incoordination, behavioral changes, decubitus, and death. One animal that died and 36 others that recovered had photodermatitis. Necropsy findings in the animals consisted of bad corporal condition, pale kidneys, evidence of liver lobular pattern, dry rumen contents, and full bladder. In two animals, fruit seeds were found in the rumen, and one animal had ulcers and transmural edema in the abomasum. Microscopically, mild to moderate renal tubular distension, accumulation of proteinaceous material in lumen with mild to moderate swelling, and epithelial necrosis. In the liver, swelling of hepatocytes and moderate bile stasis was detected. Enzymes values in all evaluated bovines were higher than those considered normal for the species. Experimentally, both calves became ill and one died. The clinical signs were apathy, inappetence, wobbling, weight loss, and goosebumps. One of them had jaundice, tearing, photophobia, ear skin detachment, and ulcers at the muzzle, nostrils and ventral face of the tongue. This animal was euthanized in extremis, and the necropsy findings showed generalized jaundice, evidence of increased liver lobular pattern, thick bile, pale kidneys, and esophageal, tongue, and epiglottal ulcers. Microscopically, the lesions were similar to those described during the natural outbreak. The skin biopsy from the calf that recovered showed perivascular edema and mild eosinophilia.

Discussion: The diagnosis was made based on clinical signs, necropsy findings, histopathological lesions, and epidemiological analysis. Experimentally, the plant was toxic at the administered doses. Photosensitization was the most common clinical sign during the natural outbreak and until now, has never been experimentally reproduced. Based on histopathological lesions observed in this study, we can consider that is from hepatogenous origin. The results showed that the kidney lesions have an important role during the pathogenesis caused by this poisoning and during disease evolution.

Keywords: toxic plants, photosensitization, ruminants, renal lesion.

Descritores: plantas tóxicas, fotossensibilização, ruminantes, lesão renal.

${ }^{1}$ Programa de Pós-graduação em Ciência Animal, Faculdade de Medicina Veterinária e Zootecnia (FAMEZ), Universidade Federal de Mato Grosso do Sul (UFMS), Campo Grande, MS, Brazil. ${ }^{2}$ Centro Universitário da Grande Dourados (Unigran), Dourados, MS. ${ }^{3}$ Programa de Pós-graduação em Ciências Veterinárias; ${ }^{4}$ Laboratório de Botânica \& ${ }^{5}$ Laboratório de Anatomia Patológica - FAMEZ, UFMS, Campo Grande. CORRESPONDENCE: R.C. Pupin [rayane.pupin@gmail.com - Fax: +55 (67) 33453615]. Programa de Pós-graduação em Ciências Veterinárias, FAMEZ - UFMS. Av. Senador Felinto Muller n. 2443. Vila Ipiranga. CEP 79074-460 Campo Grande, MS, Brazil. 


\section{INTRODUÇÃO}

O Stryphnodendron fissuratum é uma árvore nativa do cerrado pertencente a família Fabaceae e Subfamília Mimosoideae, que mede até $20 \mathrm{~m}$ de altura [14]. Por apresentar favas acastanhadas e retorcidas, é popularmente conhecida como "rosquinha" [18]. A frutificação e a queda dos frutos ocorrem durante a estação seca, entre os meses de julho e setembro $[5,6]$. A ocorrência de surtos da intoxicação pode causar importantes perdas econômicas, pois os coeficientes de morbidade e letalidade podem alcançar valores de até $16.6 \%$ e $53.3 \%$, respectivamente [5].

Os frutos são considerados a parte tóxica da planta [18], ocorrendo a intoxicação espontânea quando caem ao solo e são ingeridos pelos bovinos [5,10,11]. A intoxicação espontânea está associada a sinais clínicos digestivos, fotossensibilização e abortos em bovinos [6,19]; apenas um grupo de autores descreveu lesões renais [5].

O S. fissuratum, em experimentos, demonstrou-se tóxico para bovinos [5,18], caprinos [2,13] e porquinhos-da-índia [12], mas não reproduziu lesões de fotossensibilização em nenhuma das espécies citadas $[2,5,13,18]$.

O objetivo do presente trabalho foi descrever fotossensibilização hepatógena em bovinos causada pela ingestão espontânea e experimental de frutos de Stryphnodendron fissuratum.

\section{MATERIAIS E MÉTODOS}

\section{Surto espontâneo}

Os dados epidemiológicos e clínicos foram obtidos através da entrevista com o médico veterinário e o funcionário responsáveis pelos bovinos. Para o cálculo dos níveis de morbidade considerou-se a população exposta a planta, ou seja, os animais do lote afetado.

Para a caracterização do quadro clínico foram examinados três bovinos na fase aguda (Bovinos 1, 2 e 3 ) e outros três em fase de recuperação (Bovinos 4, 5 e 6). Amostras de sangue total para obtenção de soro foram colhidas dos bovinos na fase aguda - que foram os três animais necropsiados - e de três animais em fase de recuperação. Avaliou-se aspartato amino-transferase (AST), $\gamma$-glutamiltransferase (GGT), ureia e creatinina utilizando-se kits comerciais (Roche Cobas $\mathrm{C} 11)^{1}$. Amostras da planta foram enviadas para o setor de botânica da UFMS para identificação.
Três bovinos, todos em fase aguda, foram necropsiados e fragmentos de diferentes órgãos e também músculo esquelético foram coletados, os quais foram fixados em formol e posteriormente processados e corados rotineiramente em hematoxilina e eosina. Fragmentos de córtex, tálamo, cerebelo e medula espinhal foram coletados refrigerados e encaminhados para realização de exames de imunofluorescência direta (IFD) e inoculação intracerebral em camundongos (ICC) para diferencial de raiva.

\section{Intoxicação experimental}

Realizou-se um experimento com administração de frutos colhidos em uma fazenda localizada no Estado do Mato Grosso do Sul, na qual estavam ocorrendo casos de intoxicação por S. fissuratum em bovinos no mês de agosto. De um total de 202 bovinos, adoeceram oito e morreram quatro. Os animais recuperados manifestaram fotossensibilização.

As favas colhidas foram levadas para a Universidade Federal do Mato Grosso do Sul (UFMS) onde foram armazenadas em balcão coberto, arejado e seco, por 30 dias até o início do experimento. Amostras da planta contendo folhas e frutos foram encaminhados para a classificação no Laboratório de Botânica da UFMS.

Os animais foram alimentados com ração comercial (MACAL NUTRA 16) ${ }^{2}$, sal mineral (MACAL P68) ${ }^{2}$, água ad libitum e pastagem verde de Pennisetum purpureum (Napier) e Paspalum notatum (grama mato grosso) fornecidas no cocho trituradas.

Dois bezerros machos, mestiços, de nove meses de idade - identificados como bezerro \#1 e bezerro \#2 - foram utilizados. Os frutos foram triturados e misturados com água afim de adquirir consistência pastosa, sendo essa pasta administrada por via oral utilizando uma seringa plástica. $\mathrm{O}$ delineamento experimental está descrito na Tabela 1.

As doses utilizadas foram calculadas baseando-se em trabalhos anteriormente realizados em bovinos nos quais ocorreram quadros agudos e evolução fatal $[5,18]$. Estas doses foram reduzidas visando a reprodução de quadros com evolução prolongada, na tentativa de reproduzir a fotossensibilização.

Antes do início dos experimentos, os bezerros foram submetidos à avaliação clínica, pesagem, desverminação e coleta de sangue venoso através de punção na veia jugular para realização de hemograma e mensuração dos níveis séricos de $\gamma$-glutamiltransferase (GGT), aspartato aminotransferase (AST), ureia e creatinina 
utilizando kits comerciais (Roche Cobas C111) ${ }^{1}$. Coleta de sangue e dosagem das enzimas continuaram sendo realizadas diariamente após o início do experimento.

Fragmentos de pele foram colhidos dos bezerros \#1 e \#2, um dia antes do início da administração. Após a manifestação dos primeiros sinais de lesão cutânea - pelos arrepiados e quebradiços - novas biopsias foram realizadas na mesma região que a primeira, com proximidade de $5 \mathrm{~cm}$ da coleta anterior. Os fragmentos colhidos foram fixados em formol a $10 \%$ e processados para avaliação histológica em microscópio de luz.

Bovinos que apresentaram evolução fatal foram necropsiados, sendo coletados fragmentos de todos os órgãos que foram fixados em formol a $10 \%$ e processados rotineiramente para avaliação histopatológica.

O experimento foi aprovado pelo Comitê de Ética no Uso de Animais - CEUA da UFMS sob o protocolo $n^{\circ}$ 400/2012.

Para avaliação dos exames bioquímicos, os valores de referência considerados normais para a espécie bovina foram: (1) AST 78 - 132 U/L, (2) GGT 6.1 a $17 \mathrm{U} / \mathrm{L}$, (3) ureia 20 - $30 \mathrm{mg} / \mathrm{dL}$, (4) creatinina $1-2 \mathrm{mg} / \mathrm{dL}$ [7].

\section{RESULTADOS}

\section{Intoxicação espontânea}

O surto ocorreu em uma propriedade no município de Rio Verde do Mato Grosso (MS) que continha um rebanho total de 360 bovinos e possuía baixa oferta de forragem. O lote afetado era composto de 160 vacas e bezerros, dos quais adoeceram 52 animais, sendo 31 bezerros e 21 vacas. Destes, morreram dois bezerros e 14 vacas. O quadro clínico caracterizou-se por depressão, ataxia, incoordenação de membros pélvicos, diminuição de sensibilidade de cauda, ânus e membros pélvicos, alteração comportamental, decúbito e morte em 1 a 3 dias. Um dos animais que morreu na fase aguda desenvolveu fotodermatite, assim como os demais 36 que se recuperaram, totalizando 37 animais com lesões de fotossensibilização (Figura 1A).

Nos três bovinos necropsiados os achados macroscópicos foram semelhantes e consistiam de estado corporal ruim, rins difusamente pálidos, fígado com padrão lobular evidenciado e com múltiplos focos amarelados, conteúdo ruminal ressecado e bexiga repleta. Sementes da planta foram encontradas no rúmen de dois deles e em um dos animais (Bovino 3) havia também úlceras multifocais no abomaso e edema transmural difuso. Os achados histológicos também foram semelhantes nos três animais e caracterizaram-se, principalmente, por lesões renais e hepáticas. Nos rins havia discreta a moderada dilatação dos túbulos e acúmulo de material proteináceo eosinofílico no lúmen desses, além de discreta a moderada tumefação e necrose das células epiteliais tubulares (Figura 1B). No fígado, vacuolização moderada do citoplasma dos hepatócitos e moderada retenção biliar (Figura 1C). As lesões hepáticas lesões eram mais acentuadas no bovino 3 .

Os valores das enzimas avaliadas estavam acima dos valores de referência em todos bovinos e estão descritos na Tabela 2.

A planta foi classificada como Stryphnodendron fissuratum e foi armazenada no Laboratório de Botânica da UFMS sob o número CGMS-64898 (Figura 1D-1E).

\section{Intoxicação experimental}

Ambos os bezerros adoeceram e um morreu. Os sinais clínicos do bezerro \#1 foram icterícia leve, queda dos pelos das orelhas, apatia, andar cambaleante, diarreia, inapetência e emagrecimento. Biopsia de pele foi realizada e os achados foram edema perivascular leve e infiltrado discreto de eosinófilos. Esse animal recuperou-se.

O bezerro \#2 apresentou apatia, inapetência, andar cambaleante, emagrecimento, desidratação, salivação, lacrimejamento, secreção nasal, icterícia, fotofobia, hipotonia ruminal, diarreia, pelos arrepiados, crostas e desprendimento da pele das orelhas, úlceras no focinho, fossas nasais e face ventral da língua. $\mathrm{O}$ animal foi eutanasiado in extremis. Os achados de necropsia foram icterícia generalizada, edema na região axilar, fígado com bordos arredondados e padrão lobular evidenciado, bile espessa, rins pálidos com elevações avermelhadas, hidropericárdio, úlceras no esôfago, língua e epiglote. Microscopicamente, as principais lesões foram vistas no fígado e nos rins. No fígado, havia desorganização dos cordões de hepatócitos, tumefação hepatocelular, necrose individual aleatória e colestase leve a moderada. Nos rins, tumefação, vacuolização e necrose das células epiteliais tubulares, além de dilatação tubular e formação de cilindros hialinos. Na pele, as lesões foram características de fotodermatite e, na língua e no esôfago havia úlceras.

O hemograma dos bezerros \#1 e \#2 não apresentaram variações significativas e ocorreram elevações dos níveis séricos das enzimas avaliadas. Os valores estão expressos na Tabela 3. 
N.D. Lazaro, F.B. Bacha, R.C. Pupin, et al. 2018. Photosensitization in Cattle Caused by Spontaneous and Experimentally Ingestion of Stryphnodendron fissuratum.

\section{DISCUSSÃO}

O diagnóstico da intoxicação espontânea por Stryphnodendron fissuratum baseou-se nos sinais clínicos, achados de necropsia e histopatológico, e também na investigação epidemiológica que confirmou a escassez de pastagem, presença da planta e evidências de sua ingestão. As amostras de sistema nervoso central dos bovinos do surto natural tiveram resultado negativo para raiva. A principal condição descrita para a ingestão dos frutos é o estresse nutricional durante estação seca [12]. Experimen- talmente, o $S$. fissuratum demonstrou ser tóxico nas doses empregadas provocando a morte de bovinos com quadro clínico e patológico de insuficiência renal e hepática - com ocorrência de fotodermatite em um animal.

Sinais clínicos do surto e dos bovinos intoxicados experimentalmente foram semelhantes aos descritos em intoxicações espontâneas [5] e experimentais nessa e em outras espécies $[1,13,18]$. Outros sinais descritos como mugidos prolongados, midríase, tremores de cabeça e pescoço, timpanismo [18], fezes

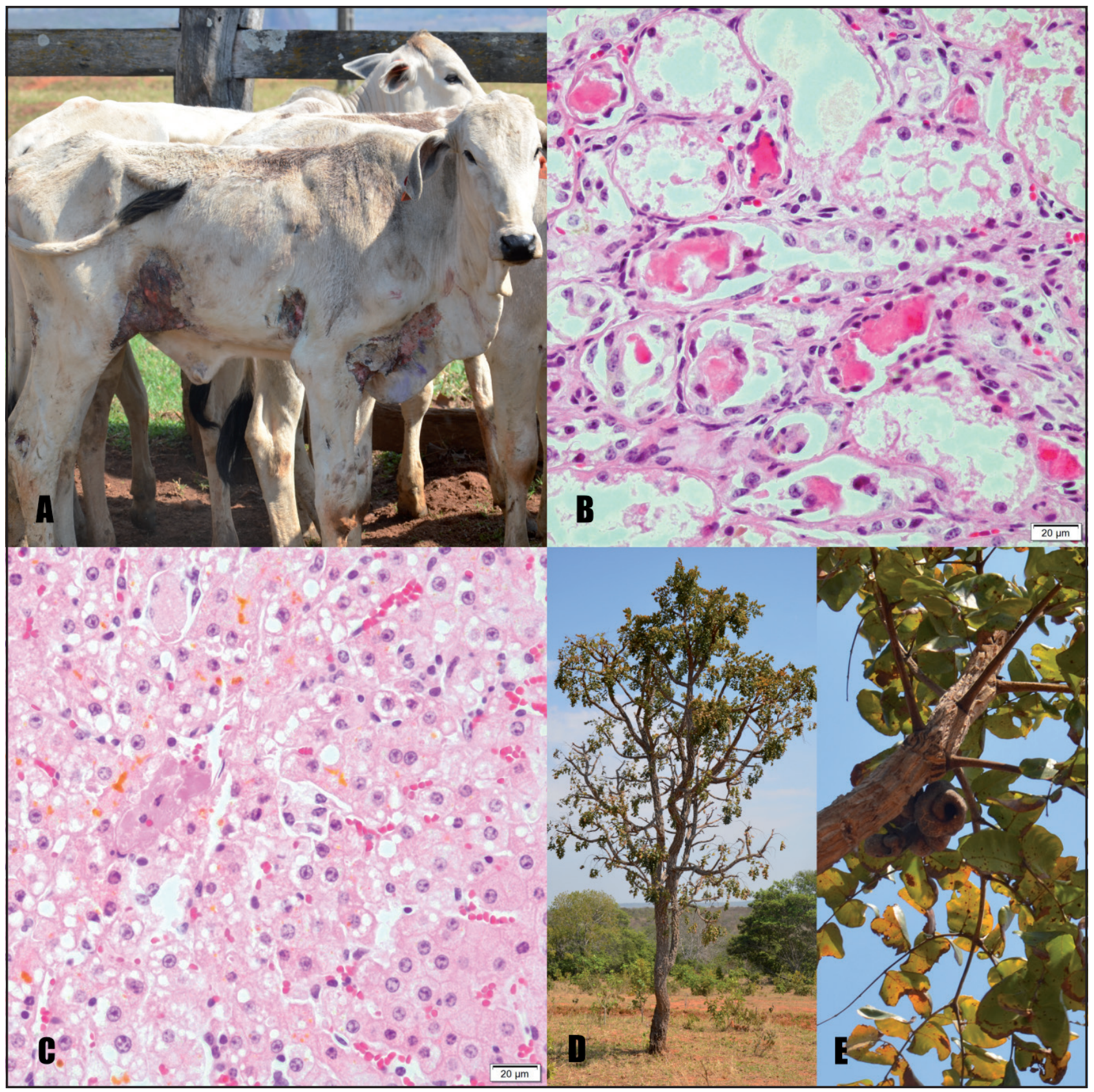

Figura 1. Intoxicação espontânea e experimental por Stryphnodendron fissuratum. A- Bezerro nelore com lesões de fotodermatite na barbela, flanco e lateral do tórax após ingestão espontânea de frutos de Stryphnodendron fissuratum. B- Intoxicação natural por Stryphnodendron fissuratum. No rim há tumefação e necrose de células epiteliais tubulares e acúmulo de material proteináceo no lúmen tubular. C- Intoxicação espontânea por Stryphnodendron fissuratum. Fígado com hepatócitos tumefeitos e moderada retenção biliar. D\&E- Árvore e frutos de Stryphnodendron fissuratum. 
Tabela 1. Intoxicação experimental por Stryphnodendron fissuratum em bezerros. Delineamento experimental - dose diária, período de administração (dias), início dos sinais clínicos e evolução.

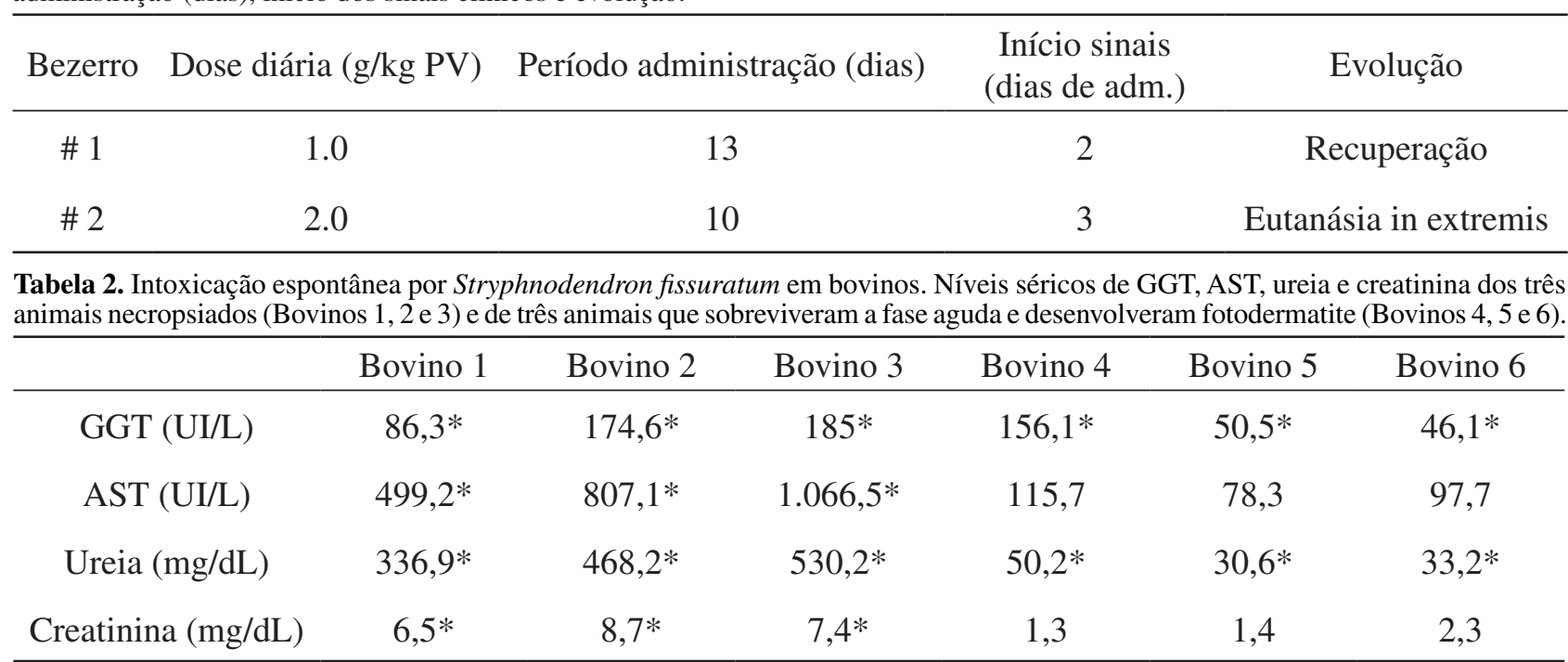

*Acima dos valores de referência.

ressequidas com muco, salivação, edema de barbela [5], regurgitação e bruxismo [5,18] não foram observados nos animais deste trabalho.

No presente estudo, o coeficiente de morbidade foi de $32,5 \%$, maior que os descritos na literatura, enquanto o de letalidade foi $30,76 \%$, menor que o máximo descrito [5]. Reproduziu-se fotossensibilização hepatógena em um dos bovinos do experimento e esta foi a manifestação observada na maioria dos animais do surto natural, ocorrendo em 71,15\% dos bovinos intoxicados. Esse sinal clínico não foi reproduzido em experimentos anteriores em bovinos [5] e caprinos $[2,13]$ e em relatos anteriores estima-se que ocorra em $20 \%$ dos animais afetados [5]. Considerando as lesões macroscópicas e histológicas no fígado observadas nos casos espontâneos [5] e experimentais [5,16] e também aquelas observadas neste estudo, pode-se considerar que a fotossensibilização na intoxicação por S. fissuratum é de origem hepatógena. A lesão cutânea ocorre nos animais que se recuperam da fase aguda da doença, caracterizada principalmente pela lesão renal, sendo semelhante ao que foi observado em um estudo com Enterolobium contortisiliquum, no qual bovinos que se recuperam da acidose ruminal desenvolveram essa manifestação [8].

Lesões na cavidade oral e no esôfago observadas no bezerro \#2 já haviam sido descritas em intoxicação experimental [1]. As lesões observadas na superfície dorsal da língua, nas gengivas e no esôfago são compatíveis com lesões extrarrenais da uremia [3], o que é fundamentado pelas lesões macro e microscópicas nos rins e também pela elevação dos valores de ureia e creatinina. Estes achados foram semelhantes aos encontrados na intoxi- cação natural por Amaranthus spinosus em bovinos [9]. Estes autores consideraram que a lesão pode estar relacionada ao efeito direto da toxina da planta sobre o órgão ou a eliminação da amônia pelo trato gastrointestinal.

As lesões macroscópicas no fígado e no rim foram semelhantes nos casos naturais e experimentais, e assemelham-se com os relatos anteriores [5,16]. Úlcera e edema na parede do abomaso foram observadas em apenas um dos animais do surto espontâneo e já foram descritas anteriormente [5,16]. Edema subcutâneo, lesões no rúmen e intestinos - áreas avermelhadas na mucosa e serosa - descritas em outros casos $[5,16]$ não foram aqui observadas. Lesões histológicas no rúmen também não foram observadas no presente estudo e são descritas por outros autores em bovinos [5,16] e caprinos [2], e são associadas a acidose ruminal [4].

Os resultados do presente estudo evidenciam que a lesão renal desempenha um importante papel na patogenia da intoxicação e na evolução da doença. Todos os animais que tiveram evolução fatal apresentaram níveis de ureia e creatinina significativamente acima dos valores fisiológicos, o que já foi descrito na literatura [17], enquanto que no bezerro 1 e nos animais do surto que se recuperaram, os níveis de ureia excederam levemente o valor de referência e a creatinina permaneceu dos valores considerados normais. As lesões renais caracterizaram-se por dilatação e necrose tubular e formação de cilindros de proteína, o que também já foi descrito em outros estudos em bovinos [1,5,16] e em caprinos [2].

As enzimas de avaliação da função hepática - AST e GGT - apresentaram marcada elevação nos casos naturais e, experimentalmente, observou-se 
níveis normais de AST e discreto aumento de GGT, embora o bovino com evolução fatal tenha desenvolvido fotodermatite e apresentasse lesões histológicas no fígado. Em estudos experimentais, AST apresentou marcada elevação e GGT moderada [17].

Apesar de não ter sido realizado o isolamento de princípios químicos no presente estudo, relatos anteriores mencionam que o princípio tóxico é desconhecido [19] mas que já foram isoladas saponinas triterpênicas, taninos hidrossolúveis, proantocianidinas e leucoantocianidinas [1,21].

Um importante diagnóstico diferencial a ser considerado, é a intoxicação por Enterolobium contortisiliquum, cujo curso clínico também está associado a abortos, fotossensibilização e sinais digestivos [20] no entanto, nessa intoxicação não são observadas lesões renais como as descritas nos casos da intoxicação pelo S. fissuratum. Nos animais que sobrevivam a fase aguda e que desenvolvem fotodermatite, deve-se diferenciar de intoxicações por plantas fotossensibilizantes, a principal delas as forrageiras do gênero Brachiaria, sendo as lesões histopatológicas suficientes para isso [15]. Nos animais que morrem de forma aguda, devido aos sinais neurológicos, deve-se diferenciar também de raiva e, nos casos que cursam com icterícia, de babesiose e anaplasmose.

\section{CONCLUSÕES}

Concluiu-se que o Stryphnodendron fissuratum é uma causa potencial de prejuízos econômicos a criação de bovinos, estando associado, principalmente, a lesões renais e hepáticas. Animais que sobrevivem a fase aguda da intoxicação podem desenvolver fotossensibilização hepatógena, sendo essa frequente. Experimentalmente, foi possível reproduzir a fotodermatite em um dos animais.

\section{MANUFACTURERS}

${ }^{1}$ Roche Diagnóstica Brasil. São Paulo, SP, Brazil. ${ }^{2}$ MACAL Nutrição Animal. Campo Grande, MS, Brazil.

Funding. This work was funded by the Fundação de Apoio ao Desenvolvimento do Ensino, Ciência e Tecnologia do Estado de Mato Grosso do Sul (FUNDECT/CNPq Grant 15/2014 PRONEM - MS).

Ethical approval. The experiment was approved by the Ethical Committee for Animal Use of UFMS under the protocol \# 400/2012.

Declaration of interest. The authors report no conflicts of interest. The authors alone are responsible for the content and writing of paper. 


\section{REFERENCES}

1 Aguiar-Filho C.R., Albuquerque R.F., Rocha B.P., Colodel E.M., Lemos R.A.A., Riet-Correa F., Evêncio-Neto J. \& Mendonça F.S. 2013. Avaliação da toxicidade das favas de Stryphnodendron fissuratum (Mimosoideae) em vacas gestantes. Pesquisa Veterinária Brasileira. 33(5): 607-612.

2 Albuquerque R.F., Evêncio-Neto J., Freitas S.H., Dória R.G.S., Saurini N.O., Colodel E.M., Riet-Correa F. \& Mendonça F.S. 2011. Abortion in goats after experimental administration of Stryphnodendron fissuratun (Mimosoideae). Toxicon. 58: 602-605

3 Breshears M.A. \& Confer A.W. 2017. The Urinary System. In: Zachary J.F. (Ed). Pathologic Basis of Veterinary Disease. 6th edn. St. Louis: Elsevier, pp.640-641.

4 Brito M.F., Tokarnia C.H. \& Peixoto P.V. 2001. Intoxicação experimental pelas favas de Stryphnodendron obovatum (Leg. Mimosoideae) em bovinos. 2 Achados ánatomo e histopatológicos. Pesquisa Veterinária Brasileira. 21(2): 61-72.

5 Ferreira E.V., Boabaid F.M., Arruda L.P., Lemos R.A.A., Souza M.A., Nakazato L. \& Colodel E.M. 2009. Intoxicação por Stryphnodendron fissuratum (Mimosoideae) em bovinos. Pesquisa Veterinária Brasileira. 29(11): 951-957.

6 Furlan F.H., Colodel E.M., Lemos R.A.A., Castro M.B., Mendonça F.S. \& Riet-Correa F. 2012. Poisonous plants affecting cattle in Central-Western Brazil. International Journal of Poisonous Plant Research. 2: 1-13.

7 Kaneko J.J. 2008. Appendix VIII Blood Analyte Reference Values in Large Animals. In: Clinical Biochemistry of Domestic Animals. 6th edn. San Diego: Academic Press, pp.882-888.

8 Leal P.V., Pupin R.C., Lima S.C., Melo G.K.A., Araújo M.A., Gomes D.C., Barros C.S.L. \& Lemos R.A.A. 2017. Ingestion of pods of Enterolobium contortisiliquum causes hepatogenous photosensitization in cattle. Toxicon. 131: 6-10.

9 Lemos R.A.A., Barros C.S.L., Salles M.S. Barros S.S. \& Peixoto P.V. 1993. Intoxicação espontânea por Amaranthus spinosus (Amaranthaceae) em bovinos. Pesquisa Veterinária Brasileira. 13(1-2): 25-34.

10 Lemos R.A.A., Jorge G.D. \& Gonçalves Júnior W. 2008. Intoxicação por Stryphnodendron fissuratum. In: Lemos R.A.A. \& Leal C.R.B (Eds). Doenças de Impacto Econômico em Bovinos de Corte - Perguntas e Respostas. Campo Grande: Editora UFMS, pp.287-289.

11 Lemos R.A.A., Guimarães E.B., Carvalho N.M., Nogueira A.P.A., Santos B.S., Souza R.I.C., Cardinal S.G. \& Kassab H.O. 2011. Plant Poisonings in Mato Grosso do Sul. In: Riet-Correa F., Pfister J., Schild A.L. \& Wierenga R. (Eds). Poisoning by Plants, Mycotoxins and Related Toxins. Wallingford: CABI Head Office, pp.68-72.

12 Macedo J.S., Rocha B.P., Colodel E.M., Freitas S.H., Dória R.G.S., Riet-Correa F., Evêncio-Neto J. \& Mendonça F.S. 2015. Congenital malformations caused by Stryphnodendron fissuratum (Leg. Mimosoideae) in guinea pigs (Cavia porcellus). Toxicon. 106: 68-71.

13 Mendonça F.S., Evêncio-Neto J., Estevão L.R.M., Melo L.E.H., Freitas S.H., Arruda L.P., Boabaid F.M. \& Colodel E.M. 2010. Aspectos clínicos da intoxicação experimental pelas favas de Stryphnodendron fissuratum (Leg. Mimosoideae) em caprinos. Pesquisa Veterinária Brasileira. 30(3): 203-210.

14 Occhioni E.M.L. 1990. Considerações taxonômicas no gênero Stryphnodendron Mart. (Leguminosae Mimosoideae) e distribuição geográfica das espécies. Acta Botânica Brasílica. 4: 153-158.

15 Riet-Correa B., Castro M.B., Lemos R.A.A., Riet-Correa G., Mustafa V. \& Riet-Correa F. 2011. Brachiaria spp. Poisoning of Ruminants in Brazil. Pesquisa Veterinária Brasileira. 31(3): 183-192.

16 Rodrigues A.S., Chaves N.S.T., Damasceno A.D., Souza M.A., Rocha Júnior L.H. \& Gonzaga Júnior W.C. 2005. Aspectos anatomo-histopatológicos da intoxicação experimental de bovinos pela ingestão de frutos de Stryphnodendron fissuratum MART. ("rosquinha”). Ciência Animal Brasileira. 6(3): 195-202.

17 Rodrigues A.S., Chaves N.S.T., Damasceno A.D., Trindade B.R., Barini A.C., Ferreira V.Q. \& Pantoja C.E.M.S. 2005. Aspectos laboratoriais da intoxicação experimental de bovinos pelos frutos de Stryphnodendron fissuratum MART. ("rosquinha"). Ciência Animal Brasileira. 6(4): 287-293.

18 Rodrigues A.S., Chaves N.S.T., Damasceno A.D., Trindade B.R., Martins G.H.L. \& Arantes A.F. 2005. Aspectos clínicos da intoxicação experimental de bovinos pelos frutos de Stryphnodendron fissuratum MART. ("rosquinha"). Ciência Animal Brasileira. 6(2): 119-126. 
19 Tokarnia C.H., Brito M.F., Barbosa J.D., Peixoto P.V. \& Döbereiner J. 2012. Stryphnodendron fissuratum. In: Plantas tóxicas do Brasil para Animais de Produção. Rio de Janeiro: Helianthus, pp.134-136.

20 Tokarnia C.H., Brito M.F., Barbosa J.D., Peixoto P.V. \& Döbereiner J. 2012. Enterolobium contortisiliquum. In: Plantas tóxicas do Brasil para Animais de Produção. Rio de Janeiro: Helianthus, pp.139-144.

21 Yokosuka A., Kawakami S., Haraguchi M. \& Mimaki Y. 2008. Stryphnosides A-F, six new triterpene glycosides from the pericarps of Stryphnodendron fissuratum. Tetrahedron. 64: 1474-1481. 